\title{
Azithromycin versus placebo for the treatment of HIV-associated chronic lung disease in children and adolescents (BREATHE trial): study protocol for a randomised controlled trial
}

Carmen Gonzalez-Martinez ${ }^{1,2,3^{*}}$, Katharina Kranzer ${ }^{4}$, Grace McHugh ${ }^{5}$, Elizabeth L. Corbett ${ }^{2,6}$, Hilda Mujuru', Mark P. Nicol ${ }^{8,9}$, Sarah Rowland-Jones ${ }^{10}$, Andrea M. Rehman ${ }^{11}$, Tore J. Gutteberg ${ }^{12,13}$, Trond Flaegstad $^{13,14}$, Jon O. Odland ${ }^{13,15}$, Rashida A. Ferrand ${ }^{5,6}$ and the BREATHE study team

\begin{abstract}
Background: Human immunodeficiency virus (HIV)-related chronic lung disease (CLD) among children is associated with substantial morbidity, despite antiretroviral therapy. This may be a consequence of repeated respiratory tract infections and/or dysregulated immune activation that accompanies HIV infection. Macrolides have anti-inflammatory and antimicrobial properties, and we hypothesised that azithromycin would reduce decline in lung function and morbidity through preventing respiratory tract infections and controlling systemic inflammation.
\end{abstract}

Methods/design: We are conducting a multicentre (Malawi and Zimbabwe), double-blind, randomised controlled trial of a 12-month course of weekly azithromycin versus placebo. The primary outcome is the mean change in forced expiratory volume in 1 second $\left(\mathrm{FEV}_{1}\right) \mathrm{z}$-score at 12 months. Participants are followed up to 18 months to explore the durability of effect. Secondary outcomes are $\mathrm{FEV}_{1} z$-score at 18 months, time to death, time to first acute respiratory exacerbation, number of exacerbations, number of hospitalisations, weight for age z-score at 12 and 18 months, number of adverse events, number of malaria episodes, number of bloodstream Salmonella typhi infections and number of gastroenteritis episodes. Participants will be followed up 3-monthly, and lung function will be assessed every 6 months. Laboratory substudies will be done to investigate the impact of azithromycin on systemic inflammation and on development of antimicrobial resistance as well as impact on the nasopharyngeal, lung and gut microbiome.

Discussion: The results of this trial will be of clinical relevance because there are no established guidelines on the treatment and management of HIV-associated CLD in children in sub-Saharan Africa, where 80\% of the world's HIVinfected children live and where HIV-associated CLD is highly prevalent.

Trial registration: ClinicalTrials.gov, NCT02426112. Registered on 21 April 2015.

Keywords: Chronic lung disease, Azithromycin, HIV, FEV 1 , Africa, Children, Obliterative bronchiolitis

\footnotetext{
* Correspondence: carmen.gonzalez@|stmed.ac.uk

${ }^{1}$ Liverpool School of Tropical Medicine, Pembroke Place, Liverpool L3 5QA, UK

${ }^{2}$ Malawi-Liverpool Wellcome Trust Clinical Research Programme, PO Box

30096, Chichiri, Blantyre 3, Malawi

Full list of author information is available at the end of the article
} 


\section{Background}

Respiratory disease is the most common manifestation of human immunodeficiency virus (HIV)/acquired immunodeficiency syndrome (AIDS) among children in sub-Saharan Africa, accounting for more than $50 \%$ of HIV-associated mortality [1-8]. The use of antiretroviral therapy (ART) and co-trimoxazole prophylaxis has contributed to a reduction in the rate of acute respiratory tract infections and mortality among HIV-infected children in both high- and low-resource settings [9].

However, studies in recent years in sub-Saharan Africa have demonstrated that about $30 \%$ of perinatally HIVinfected older children and adolescents have chronic respiratory symptoms, including chronic cough, reduced exercise tolerance and significantly impaired lung function $[4,8]$. In these studies, even participants with pronounced respiratory impairment looked well at rest, and plain radiological abnormalities were subtle. High-resolution computed tomography findings showed predominantly small airway disease consistent with constrictive obliterative bronchiolitis $(\mathrm{OB})[4,10]$. Importantly, no association was observed between abnormal lung function, ART use or duration or CD4 count, suggesting that this form of HIV-related chronic lung disease may not be responsive to ART.

$\mathrm{OB}$ is a chronic obstructive lung disease that follows a severe insult to the lower respiratory tract, resulting in fibrosis of the small airways [11]. The most common presentation is the post-infectious variant, closely related to severe viral infection in the first 3 years of life [12]. It is also seen in the context of allogeneic haematopoietic stem cell (HSC) and lung transplant recipients as a result of a graft-versus-host reaction $[13,14]$. HIV is associated with both high incidence of respiratory infections and persistent immune activation despite ART [15-17]. Thus, HIV-associated OB may share causal pathways with both post-infectious and post-transplant variants.

Evidence regarding the efficacy of treatment modalities in $\mathrm{OB}$ is sparse. Observational studies have shown some improvement of lung function in a small number of children treated with high-dose pulse corticosteroids for post-transplant OB $[14,18]$. Studies in lung transplantassociated OB have shown a positive effect of azithromycin, whereas a small randomised controlled trial of patients with established HSC-associated OB failed to show an effect $[19,20]$. Authors of case series including patients with post-infectious and post-transplant OB have reported a benefit of azithromycin on lung function and on rate of exacerbations [21].

Azithromycin is a macrolide antibiotic with bacteriostatic activity against the most common respiratory bacterial pathogens. However, it also has a robust immunomodulatory effect resulting in decreased production of proinflammatory cytokines in the acute phase and resolution of chronic inflammation in the later phases. Specifically, azithromycin has direct activity on airway epithelial cells to maintain their function and reduce mucus secretion [20, 22-24]. These characteristics have resulted in the use of azithromycin in the management of a variety of chronic lung diseases, including cystic fibrosis [25], non-cystic fibrosis bronchiectasis [26], bronchiolitis obliterans syndrome [19, 27] and chronic obstructive pulmonary disease [28]. The intracellular uptake of azithromycin is high, and hepatic excretion is slow, resulting in a long half-life that enables infrequent dosing. We will test the hypothesis that prophylactic azithromycin is effective, through its antimicrobial and anti-inflammatory properties, in preventing worsening of lung function and in reducing exacerbations in children and adolescents receiving ART who have HIVassociated chronic lung disease.

\section{Methods/design \\ Study design}

BREATHE (Bronchopulmonary function in response to azithromycin treatment for chronic lung disease in HIVinfected children) is a two-site, double-blind, placebocontrolled, individually randomised trial in which we intend to enrol 400 perinatally HIV-infected children and adolescents aged 6-19 years with HIV-associated chronic lung disease who have been receiving ART for a minimum of 6 months. Participants will be enrolled from the outpatient HIV clinics in Harare, Zimbabwe, and Blantyre, Malawi.

\section{Study intervention}

Participants are randomly assigned to receive either azithromycin or placebo in a 1:1 ratio. Weight-band azithromycin (10-19.9 kg, $250 \mathrm{mg} ; 20-29.9 \mathrm{~kg}, 500 \mathrm{mg}$; 30-39.9 kg, $750 \mathrm{mg}$; > $40 \mathrm{~kg}, 1250 \mathrm{mg} /$ week) or placebo will be given weekly under direct observation by a treatment monitor identified within the family for a total of 12 months.

\section{Study population}

HIV-infected children and adolescents with HIVassociated chronic lung disease attending outpatient HIV clinics in Harare and Blantyre will be enrolled in the trial. Individuals aged 6-19 years will be approached together with their guardians during their routine HIV outpatient visits and provided with information about the study. Once informed consent is obtained for screening, eligibility will be established using a multi-step screening procedure. Chronic lung disease will be established by spirometry (forced expiratory volume in 1 second $\left[\mathrm{FEV}_{1}\right] \mathrm{z}$-score less than -1.0$)$ with no reversibility (<12\% improvement in $\mathrm{FEV}_{1}$ after salbutamol $200 \mu \mathrm{g}$ inhaled using a spacer). Spirometry will be performed 
using the EasyOne $\mathrm{e}^{\mathrm{Tw}}$ spirometer (ndd Medical Technologies Inc., Andover, MA, USA) by trained research staff certified in performing spirometry and following the American Thoracic Society guidelines. Those who meet the criteria for chronic lung disease will undergo further tests, including a urine pregnancy test (for girls who have reached menarche), serum creatinine, alanine aminotransferase (ALT), an electrocardiogram and screening for tuberculosis (TB). For TB screening, we will use the Xpert $^{\text {tim }}$ MTB/RIF (Cepheid, Sunnyvale, CA, USA) to test one sputum sample obtained either spontaneously or through induction.

\section{Inclusion/exclusion criteria}

Perinatally HIV-infected children and adolescents aged 6-19 years who have been receiving ART for at least 6 months for HIV-associated chronic lung disease, who have a firm home address and a stable guardian, and with consent from the guardian and assent from the participant (for those aged $<18$ years; those aged $\geq 18$ years to consent independently) will be eligible for inclusion. Exclusion criteria will include having a condition that may prove fatal during the study period (e.g., malignancy), TB or acute respiratory tract infection at the time of screening, pregnancy or breastfeeding, history of cardiac arrhythmia, a prolonged QTc interval, abnormal creatinine clearance or elevated ALT, known macrolide hypersensitivity, and concomitant use of digoxin and/or fluconazole (or other drugs known to prolong the QTc interval) (Table 1).

\section{Study procedures}

Eligible individuals will be randomised to either azithromycin or placebo. The allocation ratio will be 1:1 by block randomisation with variable-length blocks stratified by site. The randomisation schedule and allocation list will be generated using Stata ${ }^{\mathrm{Tm}}$ version 14 software (2015 release; StataCorp, College Station, TX, USA) by an independent statistician not connected with the study. The allocation list will be sent directly to the study pharmacists at both trial sites, who will prepare the study medication. Participants and study personnel will therefore be masked to treatment allocation. The pharmacist will be provided with only randomly allocated numbers assigning participants to arm 1 or arm 2, with each number linked to a study number, and therefore the pharmacist will also remain blinded. It will not be possible to have pre-prepared medication packs, owing to changes of dose with weight.

After randomisation, spirometry will be repeated, and cardiopulmonary fitness will be evaluated using the incremental shuttle walk test. Transthoracic echocardiography will be performed by a trained echocardiographer using the SonoSite ${ }^{\text {tm }}$ M-Turbo echocardiography system
(FUJIFILM SonoSite, Bothell, WA, USA) at the Malawi site and the Mindray ${ }^{\text {mat }}$ DC-N6 echocardiography system (Mindray, Shenzhen, China) at the Zimbabwe site to assess right heart function and pulmonary hypertension. The research nurse will take a rectal swab, nasal aspirate, and sputum and blood samples. The blood sample will be used to measure CD4 count with the Pima ${ }^{\mathrm{ma}}$ Analyser (Alere, Orlando, FL, USA) and to perform viral load testing (Xpert $^{\text {tw }}$ HIV-1 Viral Load; Cepheid). The study drug will be dispensed by the study pharmacist. After the enrolment visit, follow-up visits will be scheduled at 2 weeks and 3-monthly thereafter. Participants will be followed for a minimum of 12 months to ascertain the primary outcome and for a further 6 months (i.e., 6 months following completion of study drug) to investigate durability of effect (if any) of the intervention. Procedures undertaken at the follow-up visits are summarised in Fig. 1.

Participants will be instructed to attend unscheduled visits if they develop acute symptoms. For specific acute symptoms, investigations and management will be as follows: for diarrhoeal episodes (Division of AIDS [DAIDS] severity grade $\geq 3$ ), a rectal swab and Clostridium difficile rapid test will be taken (C. Diff Quick Check Complete" Alere) and if positive will be treated with metronidazole for 7 days. For acute respiratory exacerbations, sputum and nasal swabs will be taken and will be treated with amoxicillin-clavulanate for 10 days; if there is no improvement, a chest radiograph and culture for TB will be obtained. For febrile episodes, blood cultures, malaria slides and or malaria rapid diagnostic tests and rectal swabs will be taken, and management will be carried out according to national guidelines. For other acute symptoms, investigations and management will be carried out at the discretion of the treating clinician. A nasal swab, rectal swab, and sputum and blood samples will be collected at study visits for measurement of immune activation markers, investigation of antimicrobial resistance and assessment of changes in the gut and respiratory microbiome.

\section{Risks}

Potential risks include an allergic reaction or adverse reaction to the medication or placebo. Examples of potential side effects include nausea, vomiting, diarrhoea and skin reactions. Each of these risks and any other unexpected outcomes will be monitored at study visits. Criteria for stopping the study drug are allergic reaction, QTc prolongation $>500$ milliseconds, hepatic toxicity with ALT $>80 \mathrm{IU} / \mathrm{L}$, concomitant use of drugs known to produce QTc prolongation, pregnancy during the course of the study and any adverse reaction with DAIDS severity grade $>3$. In case of any serious adverse events, the institutional review boards and the data and monitoring 


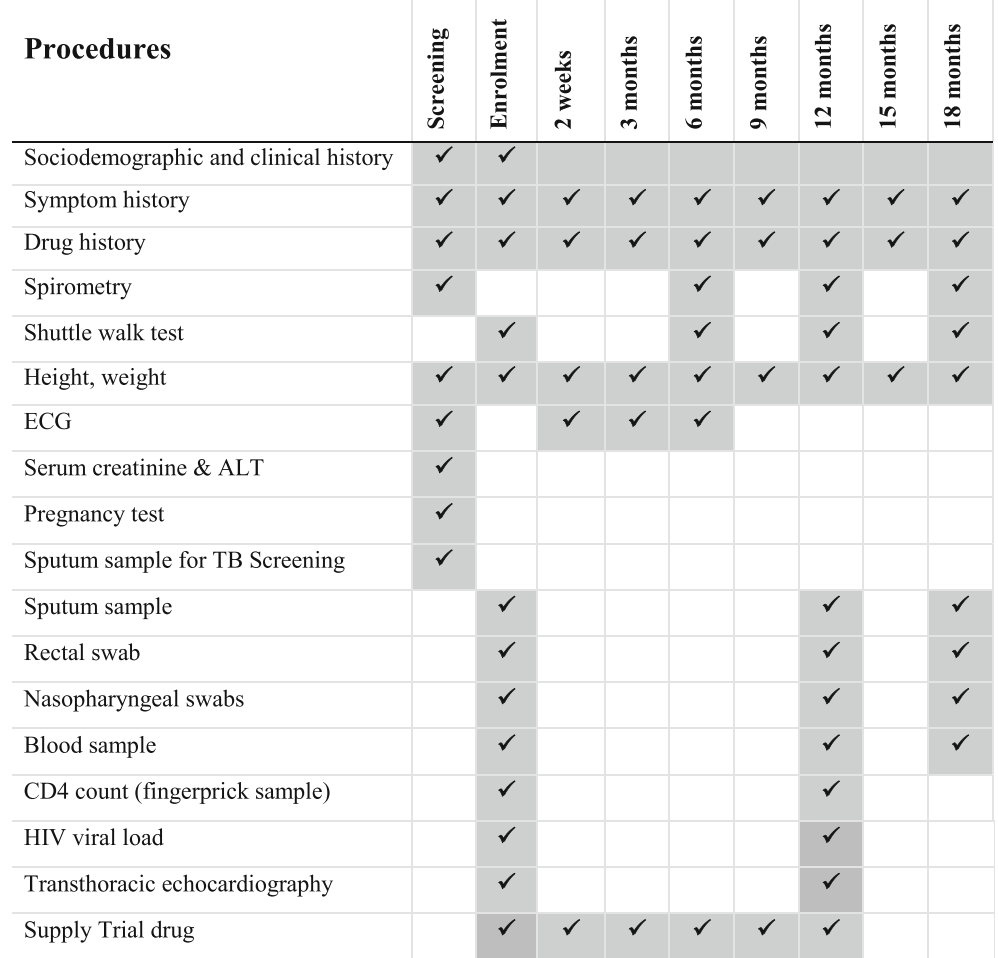

Fig. 1 Schedule of trial procedures. ALT Alanine aminotransferase, ECG Echocardiography, HIV Human immunodeficiency virus, TB Tuberculosis

safety board (DSMB) will be notified. Unblinding of treating physicians will be carried out in case of pregnancy or other events after decision by the DSMB.

\section{Outcome measures}

The primary outcome is the mean difference between trial arms in $\mathrm{FEV}_{1}$ z-scores (generated using Global Lung Function Initiative reference standards) between trial arms at 12 months after initiation of the study drug, adjusted for site and baseline $\mathrm{FEV}_{1} \mathrm{z}$-score. The secondary outcomes are described in Table 2.

Other outcomes will be the effect of azithromycin on (1) diversity and composition of respiratory and gut microbiome of children with HIV-associated chronic lung disease, (2) antibiotic resistance of bacteria colonising the respiratory tract, and (3) biomarkers of systemic inflammation.

\section{Laboratory procedures}

Nasopharyngeal and sputum samples will be frozen and stored at $-80{ }^{\circ} \mathrm{C}$ for later batch processing, which will include routine bacterial culture for respiratory pathogens, antimicrobial susceptibility testing and $16 \mathrm{~S}$ ribosomal RNA (rRNA) amplicon sequencing (microbiome analysis). Microbiome analysis of stool samples will also be done.
Archived plasma samples (isolated and stored at $-80{ }^{\circ} \mathrm{C}$ ) will be used to quantify the levels of soluble markers of immune activation and microbial translocation, including high-sensitivity $\mathrm{C}$-reactive protein, $\mathrm{D}$-dimers, interleukin6, $\beta_{2}$-microglobulin and soluble CD14 using multiplex bead assays (Luminex, Austin, TX, USA). Levels of bacterial $16 \mathrm{~S}$ rRNA will be quantified using qRT-PCR to measure the extent of microbial translocation over time. The levels of these markers will be correlated with $\mathrm{FEV}_{1} \mathrm{z}$ scores and compared between trial arms.

\section{Data collection and management and analysis plan}

Research nurses and assistants collect the data at baseline and follow-up and record it on electronic clinical record forms using Google Nexus ${ }^{\text {Tn }}$ tablets (Google, Mountain View, CA, USA) running OpenDataKit software. In both countries, data will be uploaded, processed and saved to a Microsoft Access database (Microsoft, Redmond, WA, USA) before being exported to Stata version 14.0 software and merged into a single database backed up on a regular basis. Consistency checks and checks for missing data are performed both at data entry and fortnightly once the database has been merged. Laboratory data will be recorded on paper forms and entered into the database using optical character recognition. Spirometric data will be uploaded directly from the spirometer. Echocardiographic data will be 
Table 1 Inclusion/exclusion criteria

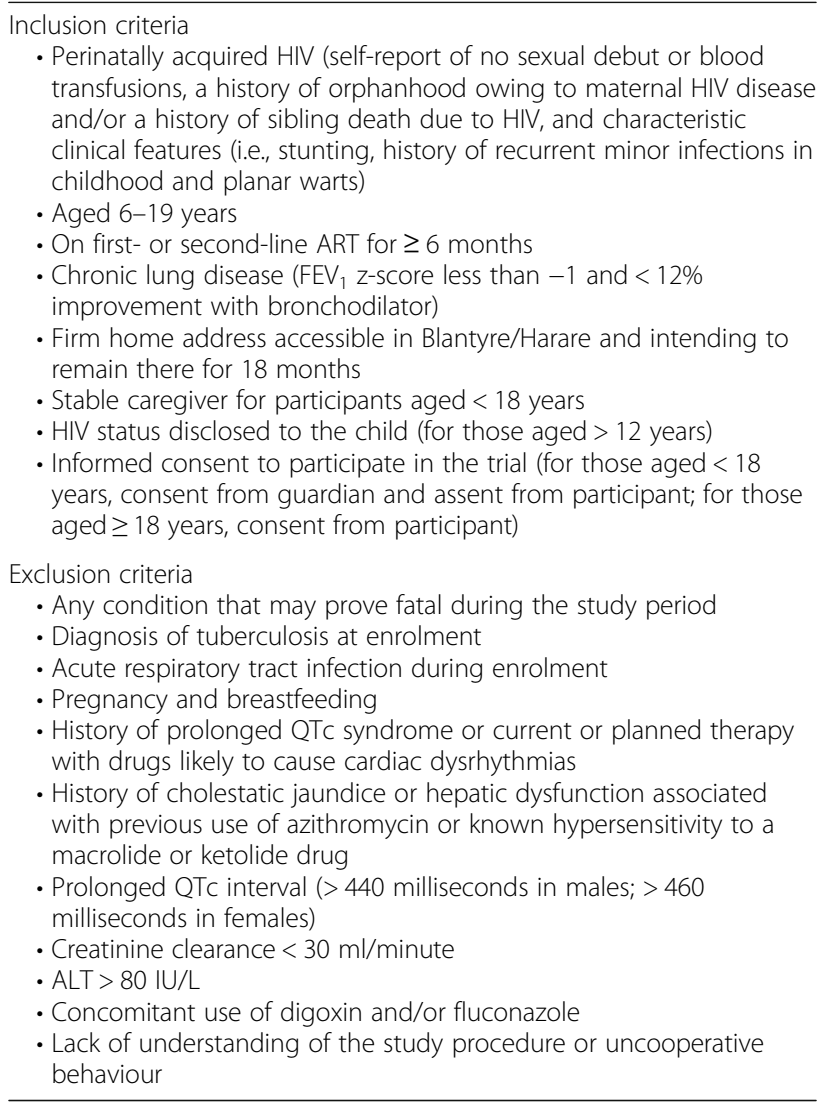

Abbreviations: ALT Alanine aminotransferase, ART Antiretroviral therapy, FEV 1 Forced expiratory volume in 1 second, HIV Human immunodeficiency virus

recorded on electronic clinical record forms, and these will be processed and saved to the Microsoft Access database for merging with the main trial database.

The primary analysis will be done on a modified intention-to-treat basis [29]. Secondary analyses will include a per-protocol analysis which will be defined using all adherence data prior to unblinding the study. Continuous outcomes will be compared between treatment

Table 2 Secondary outcomes

\begin{tabular}{ll}
\hline Outcome & Time point \\
\hline Time to first respiratory exacerbation & \\
Time to death & By 12 months \\
Number of acute exacerbations & By 12 months \\
Number of hospitalisations & By 12 months \\
$\begin{array}{l}\text { Number of mild, moderate and severe } \\
\text { adverse events }\end{array}$ & By 12 months \\
$\begin{array}{l}\text { Mean weight-for-age } z \text {-score adjusted } \\
\text { for baseline }\end{array}$ & By 12 months \\
$\begin{array}{l}\text { Incidence of infectious episodes } \\
\text { (Salmonella typhi, malaria, gastroenteritis) }\end{array}$ \\
Durability of effect (FEV ${ }_{1}$-score) & 18 months \\
\hline
\end{tabular}

FEV 1 Forced expiratory volume in 1 second groups, adjusting for site and baseline measures of the outcome and using linear regression to estimate the mean difference and corresponding 95\% CI. Time-toevent outcomes will be assessed using Cox proportional hazards regression and graphically displayed using Kaplan-Meier estimates. Between-group comparisons of binary outcomes will be analysed with logistic regression to estimate ORs and 95\% CIs. Count data (e.g., number of hospitalisations) will be analysed using Poisson regression to estimate incidence rate ratios and 95\% CIs. All analyses will be adjusted for site. Pre-specified effect modification analyses will include site and baseline severity of lung disease.

\section{Sample size and power}

The following assumptions were made to calculate the sample size:

- Mean $\mathrm{FEV}_{1}$ z-score of -2.04 in the control group

- Patients randomised in equal proportions to the two regimens

- Up to $25 \%$ of participants unassessable owing to loss to follow-up, death or suboptimal spirometric traces

- No change in $\mathrm{FEV}_{1}$ z-score in the control arm

- Difference in the trial arm mean $\mathrm{FEV}_{1}$ z-scores ranging from 0.15 to 0.3 , an effect assumed to be of clinical relevance

- SD ranging from 0.55 to 0.82 to assess the impact of variability on the difference in mean value the study has power to detect

Under these assumptions, a sample size of 400 recruited participants and 300 participants with outcome data $(25 \%$ unassessable based on previous studies of Zimbabwean children) will enable $80 \%$ power to detect a difference in trial arm means ranging between 0.17 and 0.23 , an effect size (difference in means/SD) of 0.32 (Fig. 2).

\section{Ethics and regulatory bodies}

The trial will be conducted in accordance with the principles of the Declaration of Helsinki, in compliance with the protocol approved by relevant ethics committees, and according to good clinical practice standards [30]. No change in the protocol will be implemented without the prior review and approval of the regulatory authorities, except where it may be necessary to eliminate an immediate hazard to the trial participants. Details concerning the enrolment process can be found in the Standard Protocol Items: Recommendations for Interventional Trials (SPIRIT) checklist (Additional file 1) [31].

The trial sponsor is the London School of Hygiene and Tropical Medicine (LSHTM). The trial will be monitored by the Clinical Trials Unit of the LSHTM and externally by the University of Zimbabwe Research 


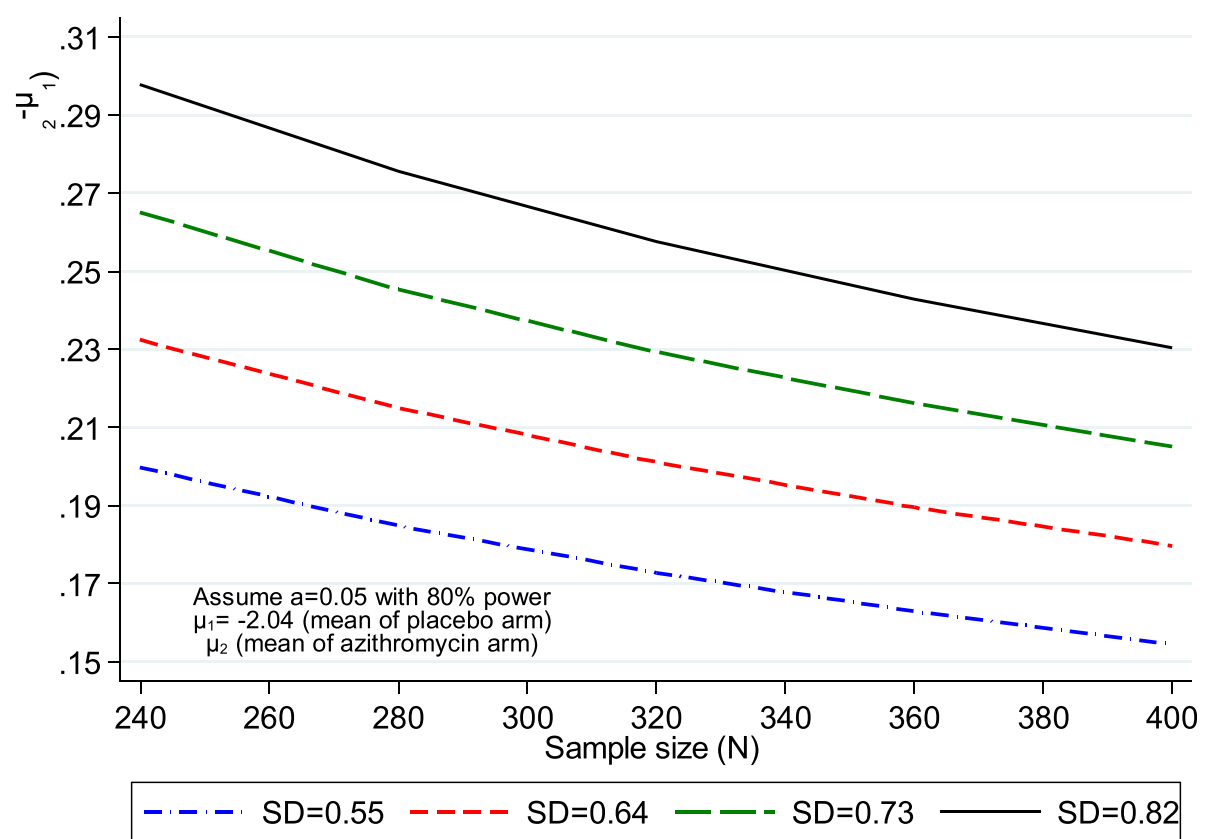

Fig. 2 Sample size calculations. FEV, Forced expiratory volume in 1 second

Support Centre in Zimbabwe and the Malawi-Liverpool Wellcome Trust Clinical Research Programme Clinical Trials Unit in Malawi.

\section{Publication}

Results of this trial will be published on completion in a peer-reviewed scientific journal. The funder will not be involved in the analysis or interpretation of the data. The full anonymised dataset will be made available no longer than 18 months after completion of the trial.

\section{Discussion}

The massive scale-up of ART programmes globally has resulted in a dramatic improvement in survival, such that increasing numbers of children perinatally infected with HIV, many of whom would have died in early childhood without HIV treatment, are now reaching adolescence. Thus, attention needs to be focused on addressing the chronic complications of long-standing HIV infection prevalent among this cohort of older HIV-infected survivors. Studies in recent years have demonstrated a high burden of chronic lung disease in children with HIV in sub-Saharan Africa, even among those receiving ART and virologically suppressed. The urgency with which evidence-based management guidelines are needed is starkly apparent: Nearly one-third of older children have chronic respiratory symptoms, and in the absence of any alternative therapeutic strategies, repeated treatment for presumptive TB is the only treatment offered and often administered. Our group has investigated bronchodilators and short course, high-dose steroids in patients with chronic lung disease who are receiving ART and isoniazid preventive therapy, with no suggestions of benefit (personal communication, T. Mwalukomo).

Whereas in the pre-ART era, lymphoid interstitial pneumonitis (LIP) was the most common cause of chronic lung disease, studies in the ART era show that $\mathrm{OB}$ is the most likely underlying cause, with LIP being an exceptional finding. $\mathrm{OB}$ is a potentially life-threatening condition that can progress to hypoxic respiratory failure and cor pulmonale and may impair lung growth in children. $\mathrm{OB}$ results from small airway inflammation and subsequent fibrosis, and in the context of HIV, it is likely that inflammation is a consequence of both HIV-mediated chronic immune activation, resulting in end-organ damage, as well as repeated infections due to HIV-mediated immunodeficiency. We therefore postulate that azithromycin is a strong candidate as a therapeutic agent for HIV-associated chronic lung disease, given its broadspectrum antibiotic activity and its anti-inflammatory and immunomodulatory properties, as well as demonstrated activity in similar chronic lung diseases and a good safety profile and tolerability. In this trial, we will investigate the effect of azithromycin on lung function as well as on acute exacerbations and other infections, and the trial will provide insight into the pathogenesis of this condition.

\section{Trial status}

The current protocol is version 2.2, dated 21 August 2017. Enrolment started in June 2016 and will continue until June 2018. 


\section{Additional file}

Additional file 1: SPIRIT checklist. (DOC $147 \mathrm{~kb}$ )

\section{Abbreviations}

AIDS: Acquired immunodeficiency syndrome; ALT: Alanine aminotransferase; ART: Antiretroviral therapy; BREATHE: Bronchopulmonary function in response to azithromycin treatment for chronic lung disease in HIV-infected children and adolescents trial; CLD: Chronic lung disease; DAIDS: Division of AIDS; DSMB: Data and safety monitoring board; ECG: Echocardiography; $\mathrm{FEV}_{1}$ : Forced expiratory volume in 1 second; HIV: Human immunodeficiency virus; HSC: Haematopoietic stem cell; LIP: Lymphoid interstitial pneumonitis; LSHTM: London School of Hygiene and Tropical Medicine; OB: Obliterative bronchiolitis; rRNA: Ribosomal RNA; SPIRIT: Standard Protocol Items: Recommendations for Interventional Trials; TB: Tuberculosis

\section{Acknowledgements}

BREATHE study team members: Tsitsi Bandason, Pauline Cavanagh, Ethel Dauya, Edith Majonga, Beauty Makamure, Gugulethu Newton Mapurisa, Slee Mbhele, Brewster Wisdom Moyo, Lucky Gift Ngwira, Jamie Rylance, Victoria Simms, Evgeniya Sovershaeva, Helen Anne Weiss, and Louis-Marie Yindom.

\section{Funding}

This trial is funded by the Global Health and Vaccination Research (GLOBVAC) Programme of the Medical Research Council of Norway.

\section{Availability of data and materials}

Datasets generated during the course of this study will be available, after analysis, in a public data repository. Before the datasets are made publicly available, all identifiable information will be removed.

\section{Authors' contributions}

JOO, RAF and ELC conceived of the study and wrote the basic protocol. JOO established the Principal Investigator's group and applied to the GLOBVAC fund on behalf of the consortium. CGM, KK, GM, ELC and HM contributed to the design and development of the study protocol. AMR led the statistical analysis with input from HAW. MPN and SRJ developed the laboratory components of the study. CGM drafted the manuscript with contributions from RAF and KK. TJG and TF were instrumental in the original protocol design and co-funding. All authors contributed to the manuscript, and all authors read and approved the final version.

\section{Ethics approval and consent to participate}

Written informed consent by the participants' guardians and assent will be obtained from participants aged younger than 18 years of age using an ageappropriate assent form before enrolment. Participants older than 18 years of age will be able to consent independently. The trial was approved by the London School of Hygiene and Tropical Medicine Ethics Committee (reference 8818) on 3 June 2015; by the Harare Central Hospital Ethics Committee on 5 October 2015; by the Medical Research Council of Zimbabwe (reference MRCZ/A/1946) on 24 May 2016; by the College of Medicine Research Ethics Committee Malawi (reference P.04/15/1719) on 7 August 2015; by the Medical Committee for Medical and Health Research Ethics, Northern Norway (reference 2015/1650) on 18 September 2015; and by the University of Cape Town Ethics Committee (reference 754/2015) on 30 June 2016. The University of Oxford did not require additional approval. Approval for conducting the clinical trial and for importation of the study investigational products was obtained from the Medicines Control Authority of Zimbabwe (26 January 2016; reference B/279/5/14/2016) and from the Pharmacy, Medicines and Poisons Board Malawi (9 March 2016; reference $\mathrm{PMPB} / \mathrm{CTRC} / \mathrm{II} / \mathrm{76}$ )

\section{Consent for publication}

Not applicable.

\section{Competing interests}

The authors declare that they have no competing interests.

\section{Publisher's Note}

Springer Nature remains neutral with regard to jurisdictional claims in published maps and institutional affiliations.

\section{Author details}

${ }^{1}$ Liverpool School of Tropical Medicine, Pembroke Place, Liverpool L3 5QA, UK. ${ }^{2}$ Malawi-Liverpool Wellcome Trust Clinical Research Programme, PO Box 30096, Chichiri, Blantyre 3, Malawi. ${ }^{3}$ Department of Paediatrics and Child Health, College of Medicine, University of Malawi, Private Bag 360, Chichiri, Blantyre 3, Malawi. ${ }^{4}$ Department of Clinical Research, London School of Hygiene and Tropical Medicine, Keppel Street, London WC1E 7HT, UK. ${ }^{5}$ Biomedical Research and Training Institute, 10 Seagrave Road, Harare, Zimbabwe. ${ }^{6}$ Department of Infectious Disease Epidemiology, London School of Hygiene and Tropical Medicine, Keppel Street, London WC1E 7HT, UK. ${ }^{7}$ Department of Paediatrics, University of Zimbabwe, PO Box A178, Avondale, Harare, Zimbabwe. ${ }^{8}$ Division of Clinical Microbiology, University of Cape Town, Anzio Road, Cape Town, South Africa. ${ }^{9}$ National Health Laboratory Service, Johannesburg, South Africa. ${ }^{10}$ Nuffield Department of Medicine, Old Road Campus, University of Oxford, Roosevelt Drive, Oxford OX3 7FZ, UK.

${ }^{11}$ MRC Tropical Epidemiology Group, London School of Hygiene and Tropical Medicine, Keppel Street, London WC1E 7HT, UK. ${ }^{12}$ Department of Microbiology and Infection Control, University Hospital of North Norway, N-9038 Tromsø, Norway. ${ }^{13}$ Faculty of Health Sciences, Arctic University of Norway, N-9037 Tromsø, Norway. ${ }^{14}$ Department of Paediatrics, University Hospital of North Norway, N-9038 Troms $\varnothing$, Norway. ${ }^{15}$ School of Health Systems and Public Health, Faculty of Health Sciences, University of Pretoria, Hatfield, South Africa.

Received: 17 September 2017 Accepted: 8 November 2017

Published online: 28 December 2017

\section{References}

1. Weber HC, Gie RP, Cotton MF. The challenge of chronic lung disease in HIVinfected children and adolescents. J Int AIDS Soc. 2013;16:18633. doi10.7448/IAS.16.1.18633.

2. Rylance J, Meghji J, Miller RF, Ferrand RA. Global considerations in human immunodeficiency virus-associated respiratory disease. Semin Respir Crit Care Med. 2016;37(2):166-80. doi:10.1055/s-0036-1572555.

3. Desai SR, Copley SJ, Barker RD, et al. Chest radiography patterns in 75 adolescents with vertically-acquired human immunodeficiency virus (HIV) infection. Clin Radiol. 2011;66(3):257-63. doi:10.1016/j.crad.2010.10.009.

4. Ferrand RA, Desai SR, Hopkins C, et al. Chronic lung disease in adolescents with delayed diagnosis of vertically acquired HIV infection. Clin Infect Dis. 2012:55(1):145-52. doi:10.1093/cid/cis271.

5. Mwalukomo T, Rylance SJ, Webb EL, et al. Clinical characteristics and lung function in older children vertically infected with human immunodeficiency virus in Malawi. J Pediatric Infect Dis Soc. 2016;5(2):161-9. doi:10.1093/jpids/piv045.

6. Rylance J, McHugh G, Metcalfe J, et al. Chronic lung disease in HIV-infected children established on antiretroviral therapy. AIDS. 2016:30(18):2795-803.

7. Githinji LN, Gray DM, Hlengwa S, Myer L, Zar HJ. Lung function in South African adolescents infected perinatally with HIV and treated long-term with antiretroviral therapy. Ann Am Thorac Soc. 2017;14(5):722-9. doi:10.1513/ AnnalsATS.201612-10180C

8. Attia EF, Weiss NS, Maleche Obimbo E, McGrath CJ, Cagle A, West TE, El Antouny NG, Attwa M, Crothers K, Chung MH. Risk factors for hypoxia and tachypnea among adolescents with vertically-acquired HIV in Nairobi. Pediatr Infect Dis J. 2017;36(4):e93-7. doi:10.1097/INF.0000000000001453.

9. Peacock-Villada E, Richardson BA, John-Stewart GC. Post-HAART outcomes in pediatric populations: comparison of resource-limited and developed countries. Pediatrics. 2011;127(2):e423-41. doi:10.1542/peds.2009-701.

10. Du Plessis AM, Andronikou S, Zar H. High resolution computed tomography for chronic small airway disease in HIV infected adolescents. Presented at the 53rd annual Pediatric Radiology Conference, Davos; 2017. http:// espr2017.org/mwg-internal/de5fs23hu73ds/progress?id=XIs7GAX9PH5860DTYU4X9joMtlwTaXbvr07B0633oM

11. Moonnumakal SP, Fan LL. Bronchiolitis obliterans in children. Curr Opin Pediatr. 2008:20(3):272-8. doi:10.1097/MOP.0b013e3282ff62e9.

12. Fischer GB, Sarria EE, Mattiello R, Mocelin HT, Castro-Rodriguez JA. Post infectious bronchiolitis obliterans in children. Paediatr Respir Rev. 2010;11(4): 233-9. doi:10.1016/j.prrv.2010.07.005. 
13. Haddad IY. Stem cell transplantation and lung dysfunction. Curr Opin Pediatr. 2013;25(3):350-6. doi:10.1097/MOP.0b013e328360c317.

14. Uhlving HH, Buchvald F, Heilmann CJ, Nielsen KG, Gormsen M, Muller KG. Bronchiolitis obliterans after allo-SCT: clinical criteria and treatment options. Bone Marrow Transplant. 2012;47(8):1020-9. doi:10.1038/bmt.2011.161.

15. Tenorio AR, Zheng Y, Bosch RJ, et al. Soluble markers of inflammation and coagulation but not T-cell activation predict non-AIDS-defining morbid events during suppressive antiretroviral treatment. J Infect Dis. 2014;210(8): 1248-59. doi:10.1093/infdis/jiu254.

16. Macatangay BJ, Yang M, Sun $X$, et al. Brief report: changes in levels of inflammation after antiretroviral treatment during early HIV infection in AIDS Clinical Trials Group Study A5217. J Acquir Immune Defic Syndr. 2017;75(1): 137-41. doi:10.1097/QAl.0000000000001320.

17. Sereti I, Krebs SJ, Phanuphak N, et al. Persistent, albeit reduced, chronic inflammation in persons starting antiretroviral therapy in acute HIV infection. Clin Infect Dis. 2017;64(2):124-31. doi:10.1093/cid/ciw683.

18. Ratjen F, Rjabko O, Kremens B. High-dose corticosteroid therapy for bronchiolitis obliterans after bone marrow transplantation in children. Bone Marrow Transplant. 2005;36(2):135-8.

19. Kingah PL, Muma G, Soubani A. Azithromycin improves lung function in patients with post-lung transplant bronchiolitis obliterans syndrome: a meta-analysis. Clin Transplant. 2014:28(8):906-10. doi:10.1111/ctr.12401.

20. Lam DC, Lam B, Wong MK, et al. Effects of azithromycin in bronchiolitis obliterans syndrome after hematopoietic SCT —a randomized doubleblinded placebo-controlled study. Bone Marrow Transplant. 2011;46(12): 1551-6. doi:10.1038/bmt.2011.1.

21. Wong C, Jayaram L, Karalus N, et al. Azithromycin for prevention of exacerbations in non-cystic fibrosis bronchiectasis (EMBRACE): a randomised, double-blind, placebo-controlled trial. Lancet. 2012;380(9842): 660-7. doi:10.1016/S0140-6736(12)60953-2.

22. Cramer CL, Patterson A, Alchakaki A, Soubani AO. Immunomodulatory indications of azithromycin in respiratory disease: a concise review for the clinician. Postgrad Med. 2017;129(5):493-9. doi:10.1080/00325481. 2017.1285677

23. Verleden GM, Vanaudenaerde BM, Dupont $\sqcup$, Van Raemdonck DE. Azithromycin reduces airway neutrophilia and interleukin-8 in patients with bronchiolitis obliterans syndrome. Am J Respir Crit Care Med. 2006;174(5):566-70.

24. Liu Y, Pu Y, Li D, Zhou L, Wan L. Azithromycin ameliorates airway remodeling via inhibiting airway epithelium apoptosis. Life Sci. 2017;170:1-8. 10.1016/.lfs.2016.11.024

25. Southern KW, Barker PM, Solis-Moya A, Patel L. Macrolide antibiotics for cystic fibrosis. Cochrane Database Syst Rev. 2012;11:CD002203. doi:10.1002/ 14651858.CD002203.pub4.

26. Valery PC, Morris PS, Byrnes CA, et al. Long-term azithromycin for indigenous children with non-cystic-fibrosis bronchiectasis or chronic suppurative lung disease (Bronchiectasis Intervention Study): a multicentre, double-blind, randomised controlled trial. Lancet Respir Med. 2013;1(8):61020. doi:10.1016/S2213-600(13)70185-1.

27. Li YN, Liu L, Qiao HM, Cheng H, Cheng HJ. Post-infectious bronchiolitis obliterans in children: a review of 42 cases. BMC Pediatr. 2014;14:238. doi:10. 1186/471-2431-14-238

28. Uzun S, Djamin RS, Kluytmans JA, et al. Azithromycin maintenance treatment in patients with frequent exacerbations of chronic obstructive pulmonary disease (COLUMBUS): a randomised, double-blind, placebocontrolled trial. Lancet Respir Med. 2014;2(5):361-8. doi:10.1016/S2213600(14)70019-0.

29. Kahan BC, Morris TP. Analysis of multicentre trials with continuous outcomes: when and how should we account for centre effects? Stat Med. 2013;32(7):1136-49.

30. International Conference on Harmonisation Guideline for International conference on harmonisation of technical requirements for registration of pharmaceuticals for human use. Guideline for Good Clinical Practice E6 (R1). ICH Harmonised Tripartite Guideline. 1996. https://www.ich.org/fileadmin/ Public_Web_Site/ICH_Products/Guidelines/Efficacy/E6/E6_R1_Guideline.pdf. Accessed 27 Mar 2017.

31. Chan AW, Tetzlaff JM, Altman DG, Laupacis A, Gøtzsche PC, Krleža-Jerić K, Hróbjartsson A, Mann H, Dickersin K, Berlin J, Doré C, Parulekar W, Summerskill W, Groves T, Schulz K, Sox H, Rockhold FW, Rennie D, Moher D. SPIRIT 2013 statement: defining standard protocol items for clinical trials. Ann Intern Med. 2013;158:200-7. doi:10.7326/0003-4819-158-3-201302050-00583.

\section{Submit your next manuscript to BioMed Central and we will help you at every step:}

- We accept pre-submission inquiries

- Our selector tool helps you to find the most relevant journal

- We provide round the clock customer support

- Convenient online submission

- Thorough peer review

- Inclusion in PubMed and all major indexing services

- Maximum visibility for your research

Submit your manuscript at www.biomedcentral.com/submit
Biomed Central 